\title{
Classical heisenberg antiferromagnet away from the pyrochlore lattice limit: entropic versus energetic selection
}

\author{
C. Pinettes \\ Laboratoire de Physique Théorique et Modélisation, Université de Cergy-Pontoise, \\ 5 Mail Gay-Lussac, Neuville-sur-Oise, 95031 Cergy-Pontoise Cedex, France \\ and 2. Physikalisches Institut, RWTH-Aachen, 52056 Aachen, Germany \\ B. Canals, C. Lacroix \\ Laboratoire de Magnétisme Louis Néel CNRS, B.P. 166, 38042 Grenoble Cedex 9, France
}

(Dated: October 25, 2018)

\begin{abstract}
The stability of the disordered ground state of the classical Heisenberg pyrochlore antiferromagnet is studied within extensive Monte Carlo simulations by introducing an additional exchange interaction $J^{\prime}$ that interpolates between the pyrochlore lattice $\left(J^{\prime}=0\right)$ and the face-centered cubic lattice $\left(J^{\prime}=J\right)$. It is found that for $J^{\prime} / J$ as low as $J^{\prime} / J \geq 0.01$, the system is long range ordered : the disordered ground state of the pyrochlore antiferromagnet is unstable when introducing very small deviations from the pure $J^{\prime}=0$ limit. Furthermore, it is found that the selected phase is a collinear state energetically greater than the incommensurate phase suggested by a mean field analysis. To our knowledge this is the first example where entropic selection prevails over the energetic one.
\end{abstract}

PACS numbers: $75.10 \mathrm{Hk}, 75.40 \mathrm{Cx}, 75.40 \mathrm{Mg}$

\section{INTRODUCTION}

In recent years, there has been a great interest in the study of fully frustrated Heisenberg antiferromagnets (HAF), hoth from the experimental and theoretical point of view 2 . These systems often show unconyentional ground-states, noncollinear, incommensurate 3 or disordered 1 . In these systems, the classical Mean Field (MF) description indicates a macroscopic ground-state degeneracy and no long range order is predicted. This property is directly related to the extensive number of de grees of freedom that still fluctuate at low temperature Nevertheless, in many cases, thermal or quantum fluctuations give rise to an entropic selection within the ground state manifold, known as "order by disorder" 6 . This phenomenon may induce a selection of a subset of the continuous degenerate ground states as it does for the kagomé antiferromagnet 7 , and sometimes can even select a particular ground state as it is the case for the face centered cubic (fcc) antiferromagnet $\mathrm{l}$.

Among these systems, the 3D pyrochlore lattice is of particular interest since it does not display such "order by disorder" and remains disordered down to zero temperature rangement of corner-sharing tetrahedra (see Fig. 1). All magnetic compounds which cristallize in the pyrochlore structure exhibit unusual magnetic properties. A few order at well defined Néel temperature 3 while many of them behave as spin glasses despite the high degree of stoichiometryt. In the Heisenberg antiferromagnetic $S=1 / 2$ quantum case, the pyrochlore lattice exhibits no magnetic transition even at $\mathrm{T}=0 \mathrm{~K}$, and behaves as a spin-liquid. The spin-spin correlation length never exceeds the inter atomic distance and it is expected that there is a fentinuum of singlet states within the singlettriplet gap 11. For classical Heisenberg spins, Monte Carlo

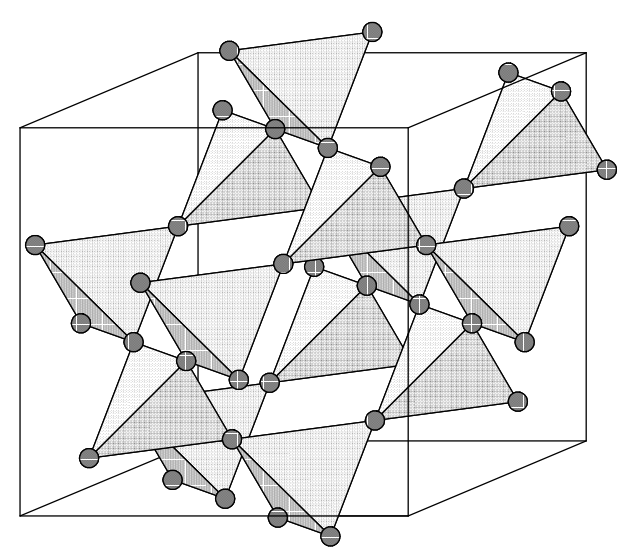

FIG. 1: The pyrochlore lattice.

simulations have shown that the pyrochlore HAF also behaves as a spin-liquid, with short ranged spin correlations both in space and time 5 国, which allows for its description to take into account only the tetrahedral unit celld 1 . The exactly solvable infinite-component spin vector model on the pyrochlore lattice also does not order down to zero temperature 22. Classical models providing much informations close to the characteristics obtained in the quantum case, it indicates that the low-energy physics in the pyrochlore structure is mainly related to the geometry of the lattice.

An interesting aspect of the problem is to study how this disordered classical ground-state resists to perturbations as it could enlarge its observation in real compounds. It has been shown that ordering is very easily induced either by including further neighbour interaction 13.14 or easy plane anisotropy 5.5 or lattice deformations 16. The purpose of this paper is to explore 
the effect of coupling the spins of the pyrochlore net to the remaining nearest-neighbor (n.n) spins in the fcc net via standard classical Monte Carlo (MC) calculations. The question is wether the system will select an ordered state when switching on this coupling or maintain disorder as in the pyrochlore HAF. As obtained in previous works, an energetic comparison of all magnetic phases at the MF level already lifts the degeneracy and strongly supports that any deviations from the pyrochlore limit will drive the system to an ordered state (sec. III). But strikingly, it is shown that thermal fluctuations do not select the expected mean field ground state but prefer a collinear phase which is more stable from the entropic point of view (sec. III]).

\section{FROM THE PYROCHLORE LATTICE TO THE FCC LATTICE : THE $J-J^{\prime}$ MODEL}

In both fcc and pyrochlore lattices, the classical energy is minimized by any configuration for which the total spin of each elementary tetrahedron in the lattice is zero. The main difference in the ground-states of these two lattices comes from the fact that in the fcc net the ordering at one tetrahedron will select a unique global ordering, while in the pyrochlore net, since the tetrahedra are more sparsely connected, there remains an extensive number of degrees of freedom 5 . In the fcc HAF, the MF ground-states are degenerate along the $\pi / a(1, q, 0)$ direction in reciprocal space. Thermal fluctuations break this degeneracy and select the collinear ordered phase $\mathbf{q}_{0}=(\pi / a, 0,0)$. These results haye been confirmed by standard Monte Carlo simulations 17 which show evidence for a first order phase transition. In the pure pyrochlore $\mathrm{HAF}$, the MF ground-states are degenerate throughout the entire Brillouin zone. Numerical works have shown that no long-range order occurs at nonzero termperature in that case, even in a regime where the temperature is below the energy scale set by interaction 5 . In between these two limits, we have considered the $J-J^{\prime}$ fcc HAF, where $J$ couples the n.n. spins on a pyrochlore net and $J^{\prime}$ the remainder n.n. spins on the underlying fcc net (see Fig. 2). This leads to the following classical Heisenberg hamiltonian :

$$
H=-J \sum_{<i j>} \mathbf{S}_{i} \cdot \mathbf{S}_{j}-J^{\prime} \sum_{<i^{\prime} j^{\prime}>} \mathbf{S}_{i^{\prime}} . \mathbf{S}_{j^{\prime}}-J^{\prime} \sum_{<i i^{\prime}>} \mathbf{S}_{i} \cdot \mathbf{S}_{i^{\prime}}
$$

where $\mathbf{S}_{i}$ is a vector spin of unit length occupying the $i^{\text {th }}$ fcc lattice site, $i$ and $i^{\prime}$ design sites on two pyrochlore nets shifted by $(2 a, 0,0)$ respectively and the sums $<>$ run over n.n. pairs. We have limited our study to the antiferromagnetic case, i.e. all negative interactions. $J=$ $J^{\prime}<0$ corresponds to the fcc HAF and $J^{\prime}=0, J<0$ leads to the pyrochlore HAF. The cubic lattice constant is $2 a$.

We have first studied the MF ground-states degeneracy for small $\alpha=J^{\prime} / J$. One way to do so is to compute the Fourier transform of the interactions on the lattice,

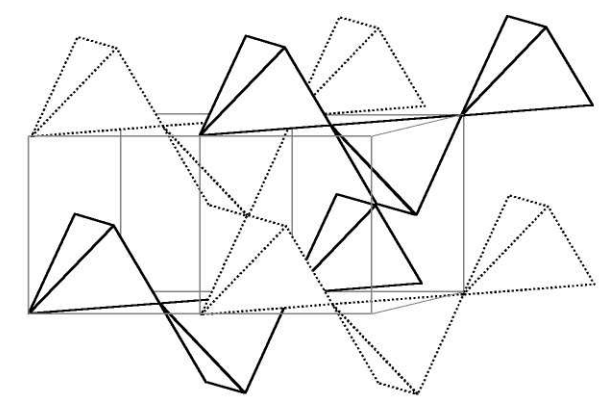

FIG. 2: The $J-J^{\prime}$ n.n. fcc lattice. The fcc lattice is decomposed into two pyrochlore nets : $A$ sites (plain lines) and $B$ sites (dashed lines). $J$ couples $A-A$ n.n. sites (plain lines) while $J^{\prime}$ couples $B-B$ n.n. sites (dashed lines) and $A-B$ n.n. sites (not drawn for clarity). This model contains 8 sites per unit cell.

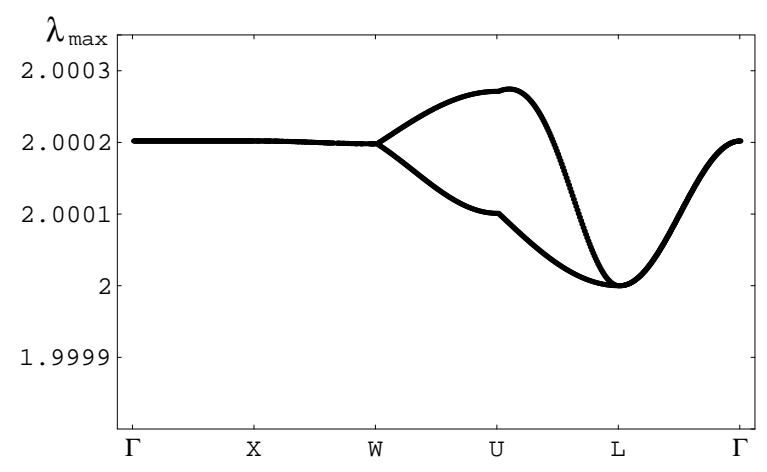

FIG. 3: Largest eigenvalue $\lambda_{\max }(\mathbf{q}) /|J|$ along the high symmetry axis of the fcc Brillouin zone for $\alpha=0.01<\alpha_{c} \approx 0.21$.

$J_{\mathbf{q}}$. The ground-state (if unique) then corresponds to the highest eigenvalue $\lambda_{\text {mat }}\left(\mathbf{q}_{1}\right)$ of $J_{\mathbf{q}}$ and is described by the propagation vector $\mathbf{q}_{1} 18$. For the $J-J^{\prime}$ model, $J_{\mathbf{q}}$ is a $8 \times 8$ matrix that has already been calculalted by Reimers et al. 13 .

For $0<\alpha<\alpha_{c} \approx 0.21$, we have reported the largest eigenvalue $\lambda_{\max }(\mathbf{q}) /|J|$ along the high symmetry axis of the fcc Brillouin zone on figure 3 . The degeneracy is completely lifted and $\lambda_{\max }(\mathbf{q})$ has an absolute maximum $\mathbf{q}_{1}$ along the $(0, q, q)$ direction, selecting an incommensurate phase of wave vector $\mathbf{q}_{1}$. Within the MF approximation the $J-J^{\prime}$ model selects an ordered phase for $0<\alpha<\alpha_{c}$.

For $\alpha \geq \alpha_{c}$, the situation is very similar to the fcc case. The MF degeneracy is lifted except on lines along $\pi / a(1, q, 0)$ direction : the system remains disordered in that case.

We have reported in figure 4 the MF energy difference $\Delta E /|J|$ between the two ground states: the $\mathbf{q}_{1}$ incommensurate state and the $\pi / a(1, q, 0)$ states (including the $\mathbf{q}_{0}$ collinear state) as a funtion of $\alpha$. We see that $\Delta E / \mid J$ is much lower than the energy scale set by the interaction $(\Delta E /|J| \sim \alpha / 100)$. Thus the degeneracy is very 


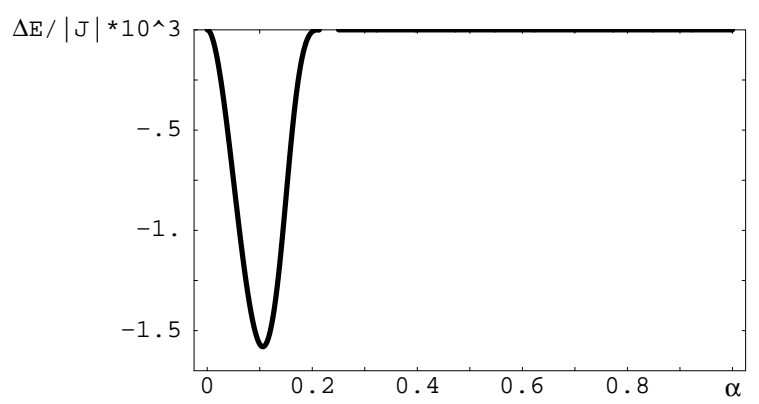

FIG. 4: MF energy difference $10^{3} \times \Delta E /|J|$ between the two $\mathrm{MF}$ ground-states as a funtion of $\alpha\left(\Delta E=E\left(\mathbf{q}_{1}\right)-E\left(\mathbf{q}_{0}\right)\right.$, see text).

slighty lifted for $\alpha<\alpha_{c}$ : the highest branch is almost flat over the whole Brillouin zone and very similar to the pyrochlore one. If we add thermal fluctuations on these MF ground-states, it is not clear what will happen. They can either select a particular state, the $\mathbf{q}_{1}$ noncollinear state (energetic selection) or a collinear state (entropic selection) or induce disorder.

\section{MONTE CARLO RESULTS}

We have performed standard MC simulations using the sample sizes of $N=864-6912$ spins with periodic boundary conditions, over a temperature range extending down to $T /|J|=10^{-4}$. We started from both random and ordered initial configurations. In each run, up to $10^{7}$ MC steps per spin have been discarded for equilibrating and up to $2 \times 10^{7} \mathrm{MC}$ steps per spins have been used for averaging. We have checked that our simulations re produce the Monte-Carloresults for the pyrochlore net: and those for the fcc net 17 . In order to determine unambigously whether the $J-J^{\prime}$ fcc HAF order or not, we have measured more specifically the specific heat per spin and a correlation function which quantify the collinearity of spins.

An unbiaised way to look for fluctuations induced order is to measure heat capacity, since it is very sensitive to the presence of zero modes (modes whose energy is independent of displacement to second order at all wave vectors 1 . From the classical equipartition theory, quadratic modes contribute a factor $T^{1 / 2}$ to the partition function $\mathrm{Z}$, while quartic modes contibute a factor $T^{1 / 4}$ : at low temperature, the behavior is thus dominated by fluctuations around the ground-states with the largest number of zero modes. These zero modes leave a signature in the heat capacity (since they contribute to $k_{B} / 4$ instead of $k_{B} / 2$ for the quadratic modes) that can be checked with Monte Carlo simulations.

In the n.n. pyrochlore HAF, fluctuations around the collinear states lead to one zero mode per tetrahedron resulting in a heat capacity per spin $C=3 k_{B} / 4$ in absence
TABLE I: Extrapolated zero temperature specific heat for various values of $\alpha=J^{\prime} / J$ from $\alpha=0$ (pyrochlore HAF) to $\alpha=1$ (fcc HAF). This system displays long range $\operatorname{order}(C=1)$ for all $\alpha>0$.

\begin{tabular}{ll}
\hline \hline$\alpha=J^{\prime} / J$ & $C$ in $\mathrm{k}_{\mathrm{B}}$ units \\
\hline 0 (pyrochlore HAF) & $0.73 \pm 0.03$ \\
0.01 & $1.01 \pm 0.02$ \\
0.1 & $1.00 \pm 0.02$ \\
1 (fcc HAF) & $0.998 \pm 0.005$ \\
\hline \hline
\end{tabular}

of order (instead of $C=5 k_{B} / 8$ if a collinear state is selected). This value has already been checked by Monte Carlo calculations 1 E. In the fcc n.n. HAF, fluctuations select the collinear state and since it contains only degeneration lines, the heat capacity per spin is simply equal to $C=k_{B}$ in that case.

As the heat capacity derived from energy fluctuations by $\mathrm{MC}$ simulations is rather noisy at low temperatures, we have estimated the heat capacity directly from the slope of the asymptotic internal energy at low temperatures. The obtained results are reported in Table [ and clearly show that for $\alpha \geq 0.01$, thermal fluctuations select an ordered state at zero temperature. To show more directly whether the system selects the collinear ordering or not, we have calculated a parameter that measures the collinearity :

$$
P\left(r_{i, j}\right)=\frac{3}{2}\left(<\left(\mathbf{S}_{i} \cdot \mathbf{S}_{j}\right)^{2}>-\frac{1}{3}\right)
$$

where $r_{i, j}$ is the distance between $i$ and $j$ sites. So $\mathrm{P}=1$ for collinear spins and $\mathrm{P}=0$ in the high temperature limit. This parameter is represented in figure 5 as a function of the separation $\mathrm{r}$ (in units of n.n. distances) at $T /|J|=$ $5 \times 10^{-4}$ for different values of $\alpha$. We find that as soon as $\alpha \geq 0.01$, the collinearity parameter is of long range, and close to 1 , contrary to the pyrochlore limit which shows a correlation for $r<2$ only. We can note that the order parameter $P(r \rightarrow \infty)$ is rather lower than 1 despite the low-temperature taken as for the XY model on the pyrochlore antiferromagnet 5 . Figure 6 shows the temperature dependence of the collinearity parameter for neighbouring spins $r=1$. As soon as $\alpha \geq 0.01$, the spins become collinear in the low temperature limit, by contrast with the pyrochlore case which has a small but finite correlation length. We have reported in figure 7 the critical temperature as a function of $\left|J^{\prime}\right|$ for $J^{\prime} \neq 0$. It is of the order of $\left|J^{\prime}\right| / 2$. We have checked that these results do not depend on the initial configuration choosen, by starting from collinear and random states. And the sizes considered are large enough since no marked finite-size effects are observed.

We have checked the low temperature phase starting from collinear and random states. We found the $\mathbf{q}_{0}$ collinear state for $\alpha \geq 0.01$. Let's notice that starting from a random configuration, stacking faults set in at low temperatures in MC calculations, as it is usual in 


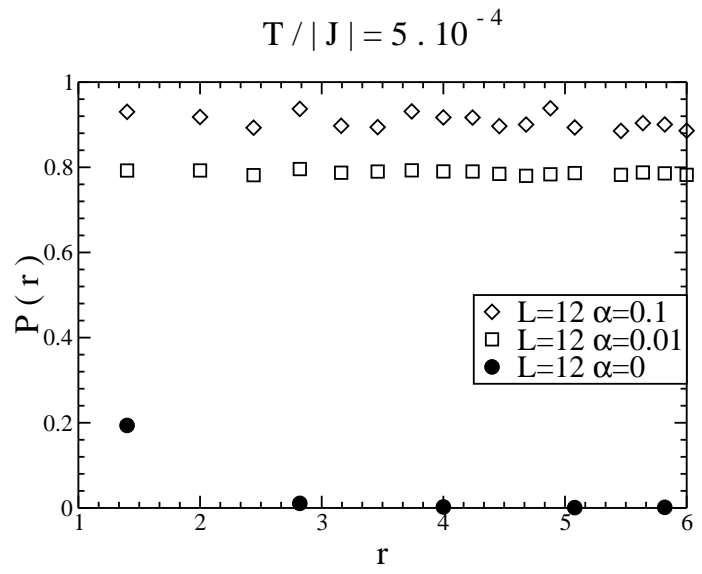

FIG. 5: Dependence of the collinearity parameter $P(r)$ at $T /|J|=5 \times 10^{-4}$ for different values of $\alpha=J^{\prime} / J, 4 \times L^{3}$, $L=12$ Heisenberg spins and starting from random spin configurations. $r$ is given in units of n.n. distances.

fully frustrated systems. At $T /|J|=5 \times 10^{-4}$ and for $\alpha=0.01$ and $N=6912$, we found two collinear blocks (of $\mathbf{q}_{0}$ type) of around $N / 2$ spins and two non collinear walls of few hundred spins. For $\alpha \geq \alpha_{c}$, we observe an order by disorder effect very similar to the fcc HAF : thermal fluctuations induces the collinear magnetic order $\mathbf{q}_{0}$ via an entropic selection. For $0<\alpha<\alpha_{c}$, the low temperature state selected by thermal fluctuations is not the $\mathbf{q}_{1}$ incommensurate state selected at the MF level, neither a state with a MF energy close to the $\mathbf{q}_{1}$ state and commensurate with the periodic boudary conditions we have taken, but the collinear state $\mathbf{q}_{0}$ whose wave vector is far from $\mathbf{q}_{1}$ in the Brillouin zone. Even for $\alpha \approx 0.1$, where the MF energy difference between these two ground states becomes greater $\left(\Delta E /|J| \sim 10^{-3}\right.$, see Fig. (1) than the lowest temperature we have considered $T /|J|=5 \times 10^{-4}$, the system still selects the collinear state. The entropic selection always overcomes the energetic one in that case.

\section{CONCLUSION}

We have studied in this paper the effect of small deviations from the pyrochlore n.n. HAF to the fcc one via standard $\mathrm{MC}$ simulations. As soon as we switch on $J^{\prime}\left(\alpha=J^{\prime} / J \geq 0.01\right)$, correlation functions are longranged in space and the specific heat at zero temperature does not indicate the presence of zero modes. The $J-J^{\prime}$ HAF selects the $\mathbf{q}_{0}=(\pi / a, 0,0)$ collinear state at low-temperature and ordering occurs at finite temperature. For small $\alpha$, thermal fluctuations do not select the MF ground-states at low temperatures but the collinear state. The entropic selection prevails over the energetic one. This is the first case, to our knowledge, where such a phenomenon is pointed out. The pyrochlore lattice

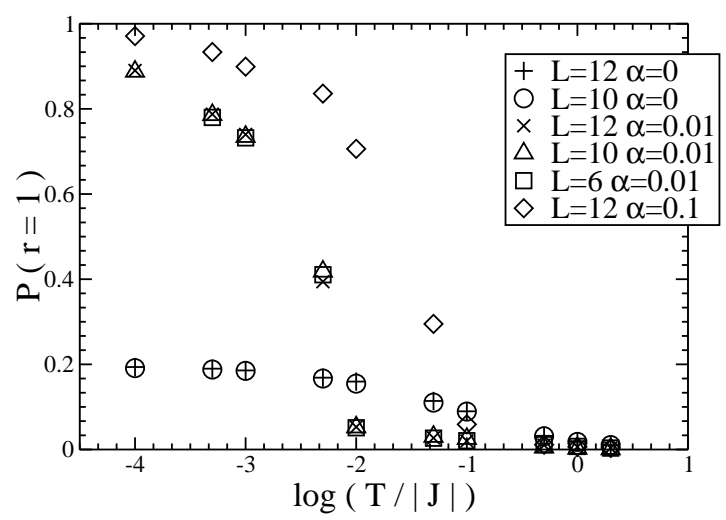

FIG. 6: Temperature dependence of the collinearity parameter $P(r=1)$ for different values of $\alpha=J^{\prime} / J$, different sizes $4 \times L^{3}$ and starting from random spin configurations.

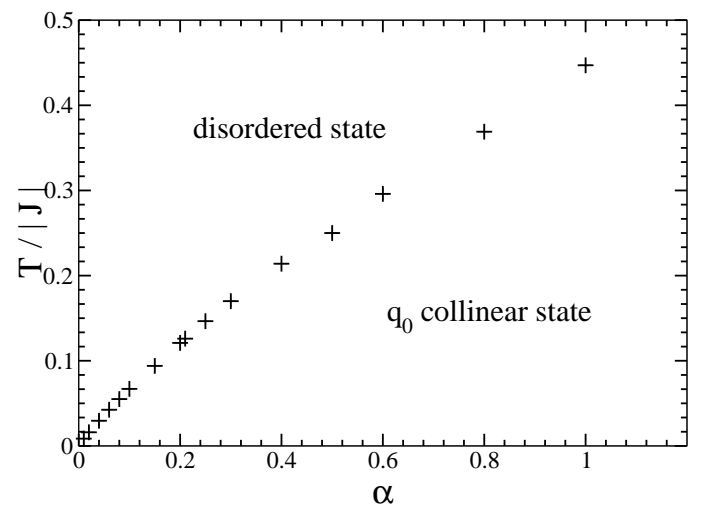

FIG. 7: Phase diagram of the $J-J^{\prime}$ model versus $\alpha=J^{\prime} / J$ $\left(\mathbf{q}_{0}=(\pi / a, 0,0)\right)$.

thus remains the only $3 \mathrm{D}$ example known with classical Heisenberg spins remaining disordered at all temperatures.

\section{Acknowledgments}

One of us (C.P.) wishes to thanks Professor H.T. Diep for helpful discussions and Professor G. Guentherodt for hospitality at the 2. Physikalisches Institut, Aachen. This work was supported in part by the DFG through SFB341. 'Laboratoire de Physique Théorique et Modélisation' is associated with CNRS (ESA 8089). 
1 P. Schiffer and A.P. Ramirez, Comments Condens. Mater. Phys. 18, 21 (1996); M.J. Harris and M.P. Zinkin, Mod. Phys. Lett. B 10, 417 (1996).

2 Magnetic Systems with Competing Interactions : Frustrated Spin Systems, edited by H.T. Diep (World Scientific, Singapore, 1994).

${ }^{3}$ G. Ferey et al., Rev. Chim. Miner. 23, 474 (1986);

${ }^{4}$ C.H. Booth et al. , Phys. Rev. B 62, R755 (2000) and references therein.

${ }^{5}$ R. Moessner and J.T. Chalker, Phys. Rev. Lett. 80, 2929 (1998); Phys. Rev. B 58, 12049 (1998).

6 J. Villain, Z. Phys. B, 33, 31 (1979); J. Villain et al., J. Phys. (Paris) 41, 1263 (1980).

7 J. T. Chalker, P. C. W. Holdsworth, and E. F. Shender, Phys. Rev. Lett. 68, 855 (1992); I. Ritchey et al., Phys. Rev. B 47, 15342 (1993)

8 C.L. Henley, J. Appl. Phys. 61, 3962 (1987); M.T. Heinila and A.S. Oja, Phys. Rev. B 48, 16514 (1993).

9 J.N. Reimers, Phys. Rev. B 45, 7287 (1992).

10 R. Moessner, A. J. Berlinsky, Phys. Rev. Lett. 83, 3293
(1999)

11 B. Canals and C. Lacroix, Phys. Rev. Lett. 80, 2933 (1998); Phys. Rev. B 61, 1149 (2000).

12 B. Canals and D.A. Garanin, cond-mat/0102237 (2001), to appear in Canadian J. Phys..

13 J.N. Reimers et al., Phys. Rev. B 43, 865 (1991).

14 J.N. Reimers et al., Phys. Rev. B 45, 7295 (1992); S.E. Palmer and J. Chalker, Phys. Rev. B 62, 488 (2000) and references therein.

15 S.T. Bramwell et al., J. Appl. Phys. 75, 5523 (1994).

16 K. Terao, J. Phys. Soc. Jpn. 65, 1413 (1996).

17 W. Minor and T.M. Giebultowicz, J. Phys. (Paris), Colloq. 49, C8-1551 (1988); H.T. Diep and H. Kawamura, Phys. Rev. B 40, 7019 (1989).

18 E.F. Bertaut, in Spin Arrangement and Crystal Structure, Domains and Micromagnets, edited by T. Rado and $\mathrm{H}$. Suhl, Magnetism Vol. III (Academic Press, New York, 1963). 